(C) 2021 IEEE. Personal use of this material is permitted. Permission from IEEE must be obtained for all other uses, in any current

or future media, including reprinting/republishing this material for advertising or promotional purposes,creating new collective

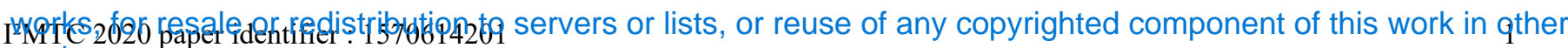
works.

\title{
Analysis of a Direct Microcontroller Interface for Capacitively-Coupled Resistive Sensors
}

\author{
Lakshmi Areekath, Student Member, IEEE, Boby George, Senior Member, IEEE, and Ferran Reverter
}

\begin{abstract}
A novel approach to directly interface a capacitivelycoupled resistive sensor to a microcontroller is presented in this paper. The existing measurement schemes for such sensors are complex. In addition, the coupling capacitance often also holds important data. The proposed simple measurement system, for such series RC sensors, is capable of measuring both the resistance and the coupling capacitance. A detailed analysis on the effect of the non-idealities on the resistance measurement showed that it is independent of the accuracy of the charging capacitor, supply voltage and preset threshold voltage. The performance of the proposed scheme has been evaluated by building suitable prototypes. Initially, a setup was designed such that the measurement was not limited by the non-idealities of the microcontroller. The test results from this showed a maximum error of $0.28 \%$ and $0.96 \%$ for the resistance and capacitance measurement, respectively. The subsequent study with the microcontroller interface exhibited a maximum error of $0.91 \%$ (resistance) and $2.94 \%$ (capacitance). Noise and resolution studies have also been conducted and the results presented. The accuracy of the prototype is promising, with a measurement time of $5 \mathrm{~ms}$ per parameter. This is a practical, low-power, low-cost measurement system as it provides digital data on the resistance and capacitance, in series, using only a microcontroller, and a couple of passive components.
\end{abstract}

Index Terms - Interfacing circuit, resistive sensor, capacitive sensor, series RC, non-contact, capacitive-coupling, direct microcontroller interface, measurement system.

\section{INTRODUCTION}

$\mathrm{C}$ APACITIVELY-coupled resistive sensors offer an efficient way to perform non-contact measurements. In these sensors, the measurement system accesses the sensing resistance through the coupling capacitances that form as a part of the sensor structure. These capacitances enable non-intrusive measurement of the quantity of interest that modulates the value of the sensing resistance. To illustrate this further, let us take the example of non-contact measurement of water conductivity, which is one of the important parameters in assessing water quality. In this, the conductivity of a water column in an insulating tube (e.g. PVC pipe) is determined without exposing the measurement electrodes to the water [1], thereby avoiding the possibility of errors in the measurement due to corrosion and formation of layers of contamination on them [2]. As illustrated in Fig. 1(a), the electrode, the insulating pipe, and the water layer directly below the electrode and in contact with the insulation form a coupling capacitance. The resistance of the water column of interest can be measured, through this coupling capacitance [1].

Resistive sensors are a popular choice to form touchpads [3], and tactile sensors [4]. Similarly, they are used to measure pressure [5], force [6], [7] and temperature [8]. Conventional resistive sensing methods require direct contact of the resistive element to the measurement circuit, thus limiting the reliability of the sensor and its versatility in the range of applications. Once a simple measurement scheme is available to read capacitively-coupled resistive sensors, some of the resistive sensors [3], [4] could be converted into capacitively-coupled ones. This will improve their reliability and the manufacturing process. Additionally, the final assembly could be simplified as the wired connection is no longer a requirement. Hence, another possible application, apart from water conductivity measurement, is in the area of resistive touchpads, wherein the change in resistance of the sensor can be measured through a capacitively-coupled arrangement. Although the focus of this paper is on measuring the resistance without direct contact between the measurement system and the resistive sensor, in certain applications, the value of the coupling capacitance also has useful information [9]. This is also addressed herein.

The schemes employed so far in the existing capacitivelycoupled resistive sensing applications [1], [10], [11] have their limitations. For instance, in [1] and [11], the circuits are relatively complex with the requirement of a precise sinusoidal excitation and a phase-sensitive detector. The need for several ICs and components for the analog and/or digital processing of the sensor signal leads to an increased power consumption. These circuit designs become complicated, mainly because the objective is to measure resistances in the range of tens of ohms $(<100 \Omega)$. Such levels of complexity cannot be justified when, for example, one aims to scale the circuit for an array of sensors, or measure higher resistance values. In fact, in the case of the conductivity measurement of water, the resistance of sea water $(55000 \mu \mathrm{S} / \mathrm{cm}[12])$ in an insulated cylindrical column of length $10 \mathrm{~cm}$ and diameter $2.5 \mathrm{~cm}$ is in the range of a few hundred ohms, but that of a drinking water outlet (diameter $1 / 4$ inch or $6.35 \mathrm{~mm}$ pipe could be sufficient) is a few hundreds of kohms. The conductivity of drinking (tap) water varies from 50$800 \mu \mathrm{S} / \mathrm{cm}$ [12]. For the capacitively-coupled measurement of higher resistances, a simpler scheme is preferred. This would be very promising in the water quality monitoring to determine the potability of drinking water [12] and the quality of river water [13], [14]. 


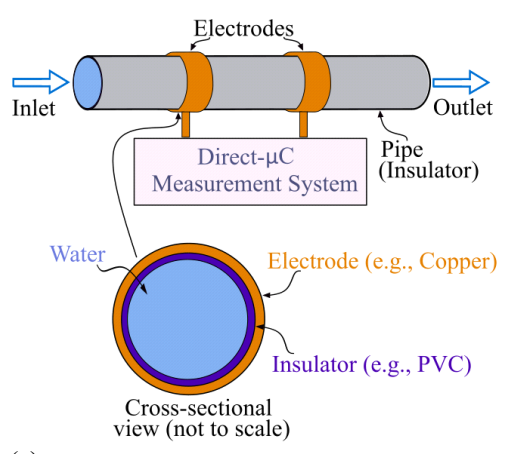

(a)

Fig. 1. (a) Illustration of non-contact conductivity measurement of water in an insulating pipe. (b) Block diagram of the proposed direct microcontroller interface for capacitively-coupled resistive sensor.

(b)
TABLE I

STATES OF THE DIGITAL PINS

\begin{tabular}{|c|c|c|c|}
\hline \hline State & DIO-1 & DIO-2 & D \\
\hline \hline 0 & LOW & LOW & LOW \\
1 & HIGH/HZ & HIGH & HZ \\
2 & LOW/HZ & LOW & HZ \\
3 & LOW & LOW & LOW \\
4 & HIGH & HZ & HZ \\
5 & LOW & HZ & HZ \\
6 & LOW & LOW & LOW \\
\hline \hline
\end{tabular}

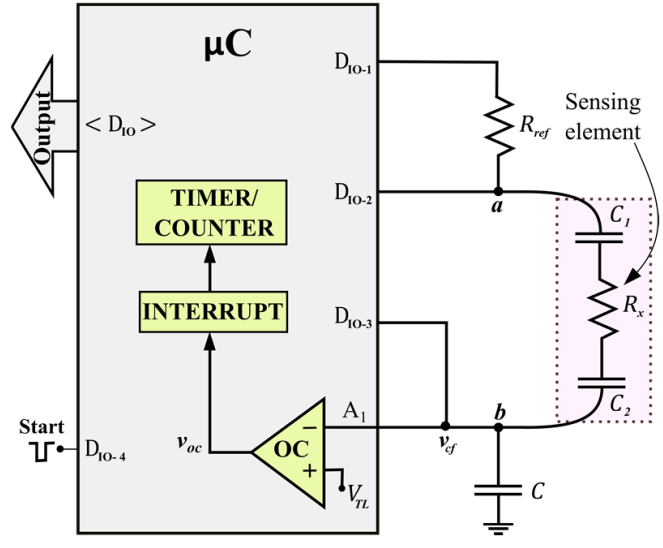

accessible for the direct connection to the $\mu \mathrm{C}$. The scheme uses a reference resistor $R_{r e f}$, and a fixed capacitor $C$. As illustrated in Fig. 1(b), the measurement can be performed using four digital input/output pins $\mathrm{D}_{\mathrm{IO}-1}, \mathrm{D}_{\mathrm{IO}-2}, \mathrm{D}_{\mathrm{IO}-3}$, and $\mathrm{D}_{\mathrm{IO}-4}$, an analog input pin $\mathrm{A}_{1}$, a timer and a comparator (OC) embedded into the $\mu \mathrm{C}$.

The two terminals of $R_{\text {ref }}$ are connected to the two digital pins $\mathrm{D}_{\mathrm{IO}-1}$ and $\mathrm{D}_{\mathrm{IO}-2}$. The terminal connected to $\mathrm{D}_{\mathrm{IO}-2}$ is also connected to node ' $a$ '. This node is connected to $C_{1}$. One end of the $C_{2}$ is accessible at node ' $b$ '. The effective capacitance $C_{12}$ due to the coupling capacitances $C_{1}$ and $C_{2}$ equals $C_{12}=$ $\left(C_{1} C_{2} /\left(C_{1}+C_{2}\right)\right)$. The fixed capacitance $C$ is connected across node ' $b$ ' and ground. The node ' $b$ ' is also connected to $\mathrm{D}_{\mathrm{IO}-3}$ and $\mathrm{A}_{1}$, as given in Fig. 1(b). $C$, along with $R_{r e f}$, and the capacitively-coupled resistive sensor, form the $\mathrm{RC}$ circuit that enables the measurement of $R_{x}$ in this direct- $\mu \mathrm{C}$ scheme. The operation of the scheme is explained below assuming ideal components. As for the microcontroller, the digital pins in $\mathrm{HZ}$ state are assumed to have an infinite input resistance, while those set as output, a zero output resistance. The non-idealities are considered and studied later in Section III.

\section{A. Measurement of $R_{x}$}

To estimate $R_{x}$, in the proposed direct- $\mu \mathrm{C}$ approach, the system operates in two modes modes based on if the sensing resistance $R_{x}$ alone is present, or the reference resistance $R_{r e f}$ is also present. Thus, the modes representing these two scenarios are, mode - $R_{x}$ and mode - $\left(R_{r e f}+R_{x}\right)$, respectively. The measurement sequence starts with mode - $R_{x}$, followed by mode - $\left(R_{r e f}+R_{x}\right)$. To execute this, the state of the pins $\mathrm{D}_{\mathrm{IO}-1}$, $\mathrm{D}_{\mathrm{IO}-2}$, and $\mathrm{D}_{\mathrm{IO}-3}$ are set as per Table-I, where LOW refers to $0 \mathrm{~V}$ or ground, HIGH to the dc voltage $V_{D D}$, and $\mathrm{HZ}$ indicates a high-impedance state. $\mathrm{D}_{\mathrm{IO}-3}$ is in $\mathrm{HZ}$ state during the charging and discharging cycles. A timing diagram of the measurement process is illustrated in Fig. 2. The measurement begins when a start pulse (high to low transition) is provided at pin $\mathrm{D}_{\mathrm{IO}-4}$. Then, the $\mu \mathrm{C}$ sets $\mathrm{D}_{\mathrm{IO}-1}, \mathrm{D}_{\mathrm{IO}-2}$, and $\mathrm{D}_{\mathrm{IO}-3}$ to $0 \mathrm{~V}$, to discharge any residual charges that may be present in the capacitances. This is indicated as state ' 0 ' in Fig. 2. Following this, $\mathrm{D}_{\mathrm{IO}-2}$ is set to HIGH, and $\mathrm{D}_{\mathrm{IO}-1}$ can be set to either HIGH or HZ. This is coupling capacitances $C_{1}$ and $C_{2}$ (refer Fig. 1(b)). As illustrated, $R_{x}, \quad C_{1}$ and $C_{2}$ are in series, and the nodes ' $a$ ' and ' $b$ ' are 


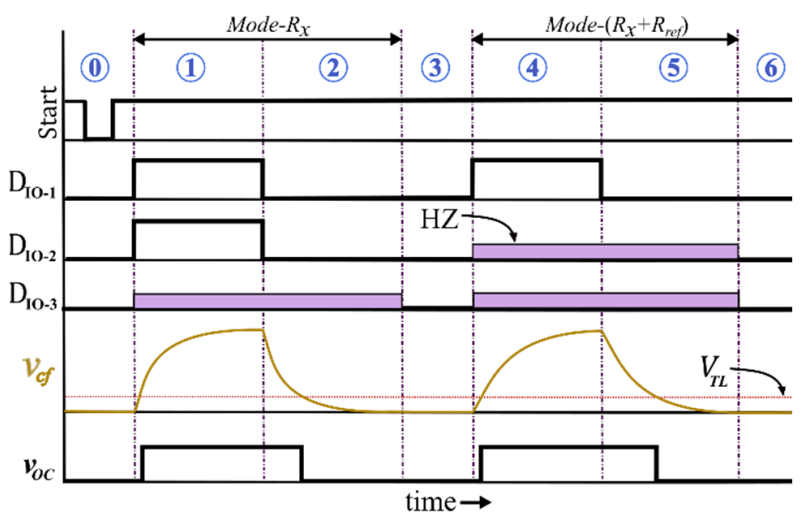

Fig. 2. Timing diagram of the proposed scheme. The signal 'start' indicates the output of $\mathrm{D}_{\mathrm{IO}-4}$.

indicated as state ' 1 ' in Fig. 2. In Fig. 2 this charging-phase is called state ' 1 '. In this phase of mode - $R_{x}, R_{x}$ along with $C_{1}$, $C_{2}$, and $C$ constitute the $\mathrm{RC}$ circuit. In this condition, $C$ is charged to the final voltage $V_{F}$ given by (1). The measurement unit is in state ' 1 ' for a preset time $T_{C h 1} . T_{C h 1}>\left(R_{x} C_{e q}\right)$, where $C_{e q}=C_{12} C /\left(C_{12}+C\right)$.

$$
V_{F}=V_{D D} \frac{C_{12}}{C_{12}+C}
$$

As soon as the time $T_{C h 1}$ is over, the state ' 1 ' ends and the state ' 2 ' begins, as in Fig. 2. In state ' 2 ', $\mathrm{D}_{\mathrm{IO}-2}$ is set to LOW, causing $C$ to discharge through $R_{x}$ as illustrated in Fig. 3(a), where $I_{d c h 1}$ indicates the associated discharging current. In state '2', $\mathrm{D}_{\mathrm{IO}-1}$ can be in either LOW or HZ states. In this discharging phase, the voltage $v_{c f}$ across $C$ is fed to the input of the comparator $\mathrm{OC}$ via $\mathrm{A}_{1}$, and compared with a preset DC threshold voltage $V_{T L}$, as illustrated in Fig. 2. The time interval $T_{1}$ taken for $v_{c f}$ to reduce from $V_{F}$ to $V_{T L}$ is measured by the embedded timer, which starts counting when the discharge begins and stops when $v_{c f}=V_{T L}$ (via interrupt to timer from OC output $v_{O C}$ ). During the discharging phase in state ' 2 ', (2) can be written when $v_{c f}=V_{T L}$. It is assumed that $V_{F}>V_{T L}$, which can be ensured, in practice, by selecting a value of $C$ such that $C<C_{12}$, and setting $V_{T L}<V_{D D} / 2$.

$$
V_{T L}=V_{F} e^{-T_{1} / R_{x} C_{e q}}
$$

From (2), we can write:

$$
T_{1}=R_{x} C_{e q} \ln \left(\frac{V_{F}}{V_{T L}}\right)
$$

The measurement unit is in state ' 2 ' for a preset time $T_{D h 1}$, where $T_{D h 1}=T_{C h 1}$. If $R_{x}$ is computed using (3), its accuracy depends on the accuracy in the measurement of $C_{e q}, V_{F}$ and $V_{T L}$. To achieve an output independent of these parameters, another mode is introduced, as explained next. Once state ' 2 ' is completed, the $\mu \mathrm{C}$ sets $\mathrm{D}_{\mathrm{IO}-1}, \mathrm{D}_{\mathrm{IO}-2}$, and $\mathrm{D}_{\mathrm{IO}-3}$ once again to LOW (like in state ' 0 '). This is indicated as state ' 3 '.

In the mode - $\left(R_{\text {ref }}+R_{x}\right)$, the RC circuit has both $R_{x}$ and $R_{\text {ref }}$ in series. $\mathrm{D}_{\mathrm{IO}-2}$ is set to $\mathrm{HZ}$ throughout this mode. $\mathrm{D}_{\mathrm{IO}-1}$ is set to HIGH to charge $C$ to $V_{F}$, and LOW for it to discharge through the same path. These are executed through the states

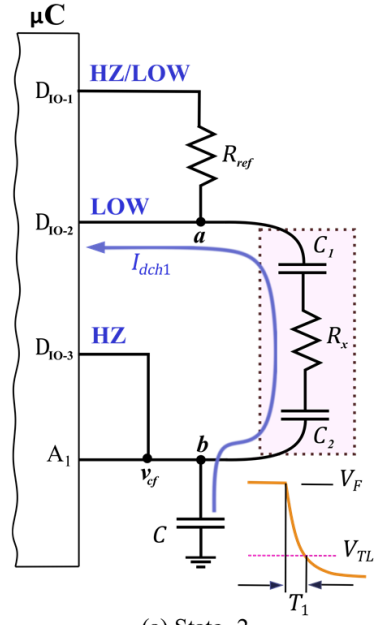

(a) State -2

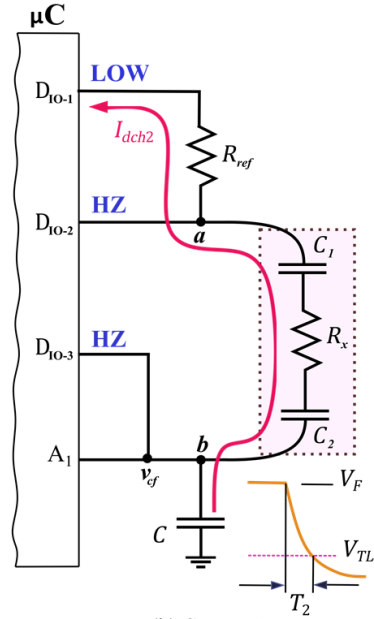

(b) State -5
Fig. 3. Discharge path of $C$ during (a) state ' 2 ', and (b) state ' 5 '.

'4' and ' 5', respectively, as illustrated in Fig. 2. The preset intervals for the states ' 4 ' and ' 5 ' are $T_{C h 2}$ and $T_{D h 2}$, where $T_{C h 2}=T_{D h 2}$ and $T_{C h 2}, T_{D h 2} \gg\left[\left(R_{x}+R_{r e f}\right) C_{e q}\right]$. As in mode - $R_{x}$, the time interval taken by $C$ to discharge from $V_{F}$ to $V_{T L}$ is measured. As illustrated in Fig. 3(b), the discharging current $I_{d c h 2}$ flows through $R_{x}, R_{r e f}$ and $C_{12}$. In this condition, the expressions for $V_{T L}$ and the corresponding discharge time, namely $T_{2}$, are given by (4) and (5), respectively. The measurement of $T_{2}$ in state ' 5 ' completes one cycle of the measurement of $R_{x}$. In case continuous measurements are to be made, the circuit operation enters state ' 6 ' once the discharge phase in mode $-\left(R_{r e f}+R_{x}\right)$ is completed. This state is identical to state ' 0 ' and facilitates the discharge of any residual charges.

$$
\begin{aligned}
& V_{T L}=V_{F} e^{-T_{2} /\left(R_{r e f}+R_{x}\right) C_{e q}} \\
& T_{2}=\left(R_{r e f}+R_{x}\right) C_{e q} \ln \left(\frac{V_{F}}{V_{T L}}\right)
\end{aligned}
$$

Once $T_{1}$ and $T_{2}$ have been measured, $R_{x}$ is estimated as follows. Dividing (5) by (3) gives (6).

$$
\frac{T_{2}}{T_{1}}=1+\frac{R_{r e f}}{R_{x}}
$$

By re-arranging (6), we get:

$$
R_{x}=\frac{R_{r e f}}{\left(T_{2}-T_{1}\right)} T_{1}
$$

Thus, the resistance $R_{x}$ of the capacitively-coupled resistive sensor can be determined in terms of the timer outputs $T_{1}$ and $T_{2}$, and the known resistor $R_{\text {ref }}$. Since $T_{1}$ and $T_{2}$ are outputs from the digital timer, the final output is digital. As can be seen from (7), the output does not depend on the value of the other parameters of the system such as $C_{1}, C_{2}, C, V_{F}$ and $V_{T L}$, or any variations of those between two consecutive measurements of $R_{x}$. However, the measurement output does depend on $R_{r e f}$. For this reason, such a reference component should be selected with a low tolerance and a low thermal sensitivity. 


\section{B. Measurement of $C_{12}$}

In certain applications, the value of the effective coupling capacitance $C_{12}$ carries useful information. For example, in the case of resistance measurement of the water column in pipes, the actual inner diameter of the pipe will change over long time, due to deposition of organic/inorganic particles and microorganisms. Thus, the value of $R_{x}$ will be higher, for the same conductivity, compared to that measured with the original dimensions of the pipe. In such a scenario, the measurement of the coupling capacitance $C_{12}$ can provide information about these inner layers formed and, hence, corrective measures can be taken. Hence, it would be beneficial if the proposed direct microcontroller interface could be extended to determine the value of $C_{12}$. Such a method can also be used to measure the capacitance value, in addition to the resistance value, in lossy capacitive sensors where the resistance and capacitance are in series, in the electrical equivalent circuit.

To measure $C_{12}$, the same two modes of operation, mode $R_{x}$ and mode - $\left(R_{\text {ref }}+R_{x}\right)$ are used. The only difference is that in each mode an additional measurement corresponding to a different threshold $V_{\text {TLnew }}$ is made, i.e. in each mode, two measurements are made, namely, one corresponding to $V_{T L}$ and the other to $V_{\text {TLnew }}$, where $V_{\text {TLnew }}<V_{T L}$. This is illustrated in Fig. 4.

In mode - $R_{x}$, the two discharging times to be measured are: $T_{1}$ for $C$ to discharge to $V_{T L}$, and $T_{1 \text { new }}$ for $C$ to discharge to $V_{\text {TLnew }}$. To determine $T_{1 \text { new }}, V_{T L}$ and $T_{1}$ in (2) are replaced with $V_{\text {TLnew }}$ and $T_{1 \text { new }}$, respectively:

$$
V_{\text {TLnew }}=V_{F} e^{-T_{1 \text { new }} / R_{x} C_{e q}}
$$

Dividing (2) by (8), taking logarithm and re-arranging, we get:

$$
T_{1 \text { new }}-T_{1}=R_{x} C_{e q} \ln \left(\frac{V_{T L}}{V_{T L n e w}}\right)
$$

Similarly, in mode - $\left(R_{r e f}+R_{x}\right)$, in addition to measuring the discharging time $T_{2}$ from the decaying exponential curve given in (4), $T_{2 \text { new }}$ is measured based on (10).

$$
V_{\text {TLnew }}=V_{F} e^{-T_{2 n e w} /\left(R_{r e f}+R_{x}\right) C_{e q}}
$$

Further, from (4) and (10), (11) is arrived at, as shown below.

$$
T_{2 n e w}-T_{2}=\left(R_{\text {ref }}+R_{x}\right) C_{e q} \ln \left(\frac{V_{T L}}{V_{T L n e w}}\right)
$$

The selection of the threshold voltages $V_{T L}$ and $V_{\text {TLnew }}$ must be done such that,

i) The difference between $V_{T L}$ and $V_{T L n e w}$ is much higher than the typical noise level in both the signal $v_{c f}$ and the input of the comparator.

ii) The difference in the measured time coming from (9) and (11) is much greater than the resolution of the timer used.

Let $T_{1 \text { new }}-T_{1}=\Delta T_{1}$ and $T_{2 n e w}-T_{2}=\Delta T_{2}$. Then, the difference between (11) and (9) gives (12).

$$
\Delta T_{2}-\Delta T_{1}=R_{\text {ref }} C_{e q} \ln \left(\frac{V_{T L}}{V_{T L n e w}}\right)
$$

From (12),

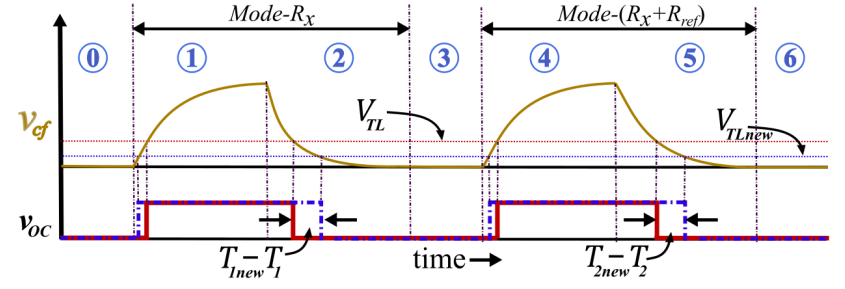

Fig. 4. Timing diagram illustrating the $v_{c f}$ and $v_{O C}$ waveforms, in Mode- $R_{x}$ and Mode- $\left(R_{x}+R_{\text {ref }}\right)$, using two threshold voltages $V_{T L}$ and $V_{T L n e w}$.

$$
C_{e q}=\frac{\Delta T_{2}-\Delta T_{1}}{R_{r e f} \ln \left(\frac{V_{T L}}{V_{T L n e w}}\right)}
$$

Thus, using the value of $C_{e q}$ obtained from (13), the value of $C_{12}$ can be obtained using (14).

$$
C_{12}=\frac{C_{e q} C}{C-C_{e q}}
$$

The above method to measure $C_{12}$ is a simple, yet efficient one. According to (13), $C_{e q}$ and hence $C_{12}$ depend on $V_{T L}$ and $V_{\text {TLnew }}$. A one-time correction to reduce the effect of the inaccuracy of $V_{T L}$ and $V_{T L n e w}$ in $C_{12}$ measurement is discussed in section III. A.

\section{ERROR ANALYSIS}

The important sources of error and their effects on the final output are discussed in this section. The non-idealities of the comparator, digital pins, accuracy of threshold voltages, and resolution of the timer are considered for the analysis.

\section{A. Input Offset Voltage of Comparator and Threshold Voltage}

Let $V_{O S}$ be the input offset voltage of the comparator OC. In its presence, (3) and (5) become (15) and (16), respectively.

$$
\begin{aligned}
& T_{1}=R_{x} C_{e q} \ln \left(\frac{V_{F}}{V_{T L \pm V}}\right) \\
& T_{2}=\left(R_{r e f}+R_{x}\right) C_{e q} \ln \left(\frac{V_{F}}{V_{T L} \pm V_{O S}}\right)
\end{aligned}
$$

Dividing (16) by (15) gives the same equation as (6), from which $R_{X}$ can be obtained as in (7). Thus, the presence of $V_{O S}$ has no impact on the determination of $R_{x}$ using the proposed scheme.

The same analysis holds good for the effect of variation in the threshold voltage. As can be seen in (15) and (16), any change in the threshold voltage comes as an addition or subtraction of $V_{O S}$. Since the measurement of $R_{x}$ is not affected by the presence of $V_{O S}$, the inaccuracy of the threshold voltage does not affect the accuracy of the final output provided that it is stable within one measurement cycle.

As for the measurement of $C_{12},(12)$ becomes (17).

$$
\Delta T_{2}-\Delta T_{1}=R_{r e f} C_{e q} \ln \left(\frac{V_{T L} \pm V_{O S}}{V_{T L n e w} \pm V_{O S}}\right)
$$

The resulting capacitance obtained herein can be termed as $C_{e q}^{\prime}$, and is given by (18). The corresponding coupling capacitance $C_{12}^{\prime}$ is given in (19). 


$$
\begin{aligned}
C_{e q}^{\prime} & =\frac{C_{e q}}{1+\epsilon} \\
C_{12}^{\prime} & =\frac{C_{e q} C}{C(1+\epsilon)-C_{e q}}
\end{aligned}
$$

In (18), $C_{e q}$ is the capacitance in the absence of $V_{O S}$ given by (13), and $\epsilon=\ln \left[\frac{1 \pm V_{O S} / V_{T L}}{1 \pm V_{O S} / V_{T L n e w}}\right] / \ln \left(V_{T L} / V_{T L n e w}\right)$. For a typical $V_{O S}$ of $10 \mathrm{mV}$, and threshold voltages of $V_{T L}=500 \mathrm{mV}$ and $V_{\text {TLnew }}=300 \mathrm{mV}$, then $\epsilon=-0.02$. The value of $(1+\epsilon)$ can be estimated by performing an one-time measurement using a known value of $C_{e q}$. In the later measurements, the actual $C_{e q}$ can be calculated as $C_{e q}^{\prime}(1+\epsilon)$, and the same can be used to estimate $C_{12}$ as in (14).

As mentioned above, the effect of change in the threshold voltage can be represented as an equivalent $V_{O S}$. Therefore, when we compensate for the effect of $V_{O S}$ in the $C_{12}$ measurement, the effect due to inaccuracy of the threshold voltages will also get corrected.

\section{$B$. Variation in the Voltage $V_{D D}$}

The de voltage $V_{D D}$ provided by a digital pin in HIGH state is not derived from a precision voltage source. Hence, variations are expected in it, e.g., $V_{D D} \pm \Delta v_{d d}$, where $\Delta v_{d d}$ is the variation. As one cycle measurement is completed in a very short time, $\Delta v_{d d}$ is assumed constant during that cycle. In such a case, as $V_{F}=\left(V_{D D} \pm \Delta v_{d d}\right) C_{12} /\left(C_{12}+C\right)$, (3) and (5) become (20) and (21), respectively.

$$
\begin{aligned}
& T_{1}=R_{x} C_{e q} \ln \left[\frac{\left(V_{\left.D D \pm \Delta v_{d d}\right) C_{12}}\right.}{V_{T L}\left(C_{12}+C\right)}\right] \\
& T_{2}=\left(R_{r e f}+R_{x}\right) C_{e q} \ln \left[\frac{\left(V_{D D \pm} \pm \Delta v_{d d}\right) C_{12}}{V_{T L}\left(C_{12}+C\right)}\right]
\end{aligned}
$$

As $\ln \left[\frac{\left(V_{D D \pm} \pm v_{d d}\right) C_{12}}{V_{T L}\left(C_{12}+C\right)}\right]$ is present in both the terms, (6) and the final output as per (7) are not affected.

As for the measurement of $C_{12}$, the variation in $V_{D D}$ is irrelevant since the time differences as per (9), (11) and $C_{12}$ as per (13) do not have the term $V_{D D}$.

\section{Internal Resistance of the Digital Pins}

Digital pins offering zero output resistance was assumed while explaining the operation in section II. To analyze the impact of the internal resistance $R_{i}$ of the digital pin in the measurement of $R_{x}$ and $C_{12}$, it is assumed that identical $R_{i}$ is present at each digital input pin. In reality, there may be a mismatch of about a tenth of an ohm.

In the presence of $R_{i}$, the discharge time constant in mode$R_{x}$ is modified to $\left(\frac{R_{i}\left(R_{r e f}+R_{i}\right)}{R_{r e f}+2 R_{i}}+R_{x}\right) C_{e q}$. However, since $\frac{R_{i}\left(R_{r e f}+R_{i}\right)}{R_{r e f}+2 R_{i}} \ll R_{x}$ in the prototype, the effects of $R_{i}$ on the time measurement are expected to be negligible. In mode - $\left(R_{r e f}+\right.$ $R_{x}$ ), the discharge time constant becomes $\left(R_{\text {ref }}+R_{x}+R_{i}\right) C_{e q}$. Again, since $R_{i} \ll\left(R_{\text {ref }}+R_{x}\right)$, it can be neglected and thus does not significantly impact the time measurement. On the other hand, as long as the charging time is sufficiently longer (at least, five times higher) than the charging time constant, the charging phase is negligibly affected by $R_{i}$. Consequently, in the prototype developed, the measurement of $R_{x}$ and $C_{12}$ should not be affected by $R_{i}$.

\section{Parasitic Capacitance of the Digital pins}

Each digital pin, together with the PCB trace or cable to the sensor introduces a parasitic capacitance from the pin to ground. The capacitances $C_{s 1}, C_{s 2}$, and $C_{s 3}$, shown in Fig. 5, represent these parasitic capacitances, respectively, from node $\mathrm{D}_{\mathrm{IO}-1}, \mathrm{D}_{\mathrm{IO}-2}$, and $\mathrm{D}_{\mathrm{IO}-3}$ to ground. As these parasitic capacitances are in the range of a few $\mathrm{pF}$, and the output resistance of the digital pins is in the range of tens of ohms, the discharge-time measurements in the mode- $R_{x}$ have negligible impact due to $C_{s 1}$ and $C_{s 2}$. As $C_{s 3}$ is in parallel to $C$, the effective $C$ will become $C+C_{s 3}$, and $C_{e q}$ will get modified as $C_{e q}^{s 3}=\frac{C_{12}\left(C+C_{s 3}\right)}{C_{12}+C+C_{s 3}}$. Since $C_{e q}$ is not present in the estimation of $R_{x}$ as in (7), there will be no effect.

In the case of mode- $\left(R_{r e f}+R_{x}\right)$, there are two discharging events happening simultaneously: (a) discharging of $C_{s 2}$ through $R_{\text {ref }}$ to ground, and (b) discharging of $C_{e q}^{s 3}$ through $\left(R_{\text {ref }}+R_{x}\right)$. Since the time constant $C_{s 2} R_{\text {ref }} \ll C_{e q}^{s 3}\left(R_{\text {ref }}+\right.$ $R_{x}$ ), it does not affect the discharging along the main discharge path (b). To keep the effect of $C_{s 3}$ very low, the condition $C_{s 3} \ll$ $C$ should be ensured. As $C$ is in $\mathrm{nF}$ and $C_{s 3}$ is in $\mathrm{pF}$, the effect in $C_{e q}$ and $C_{12}$ is expected to be negligible.

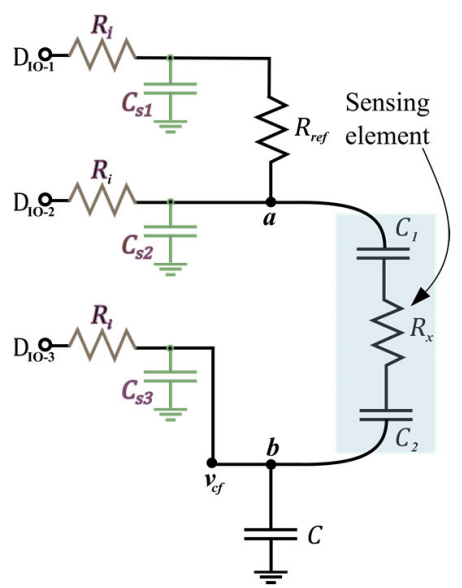

Fig. 5. The parasitic capacitances $C_{s 1}, C_{s 2}$, and $C_{s 3}$ at the digital input pins.

\section{E. Resolution of the Timer}

The resolution of the timer embedded into the microcontroller plays an important role in the final accuracy. In the prototype developed, the timer has a resolution of $62.5 \mathrm{~ns}$, and the time interval under measurement is in the range of a few hundred microseconds for the $R_{x}$ measurement. In the case of $C_{12}$ measurement, the time difference estimated as per (9), (11) is in the range of tens of microseconds. Therefore, the limited resolution of the timer will have more effect on the accuracy of the $C_{12}$ measurement. To achieve a higher accuracy, a timer with a higher resolution is recommended.

\section{EXPERIMENTAL SETUP AND RESUlts}

The hardware prototype of the proposed scheme, presented in Fig. 1(b), was realized in two steps, for the measurement of both $R_{x}$ and $C_{12}$. In the first step, a prototype was developed to 
test the feasibility of the approach without using a microcontroller. For this, a data acquisition system (DAS), namely Analog Discovery kit (14-bit resolution, $100 \mathrm{MS} / \mathrm{s}$ sampling rate) from Digilent, and a Virtual Instrument (VI), developed using NI LabVIEW, were used. The function generator, included in the DAS, was programmed to generate the HIGH and LOW state signals as required in the two modes of measurement given in Table-I and Fig. 2. As indicated in Table-I, DIO-1 can be in HZ/high in state-1, and HZ/low in state2. During the experimental tests it was kept in high during state1 and low in state-2. In this DAS, the operation of the function generator is independent of the other operations, e.g., digitization, data transfer, etc. Thus, the signal at node ' $b$ ' was digitized using the DAS and analyzed, as described below. In the second step, a microcontroller-based prototype was developed and tested. In this case, the two modes of measurement were realized by setting the microcontroller pins in accordance with Table-I and Fig. 2. The components used to build the prototypes are listed in Table - II. A photograph of the experimental setup is shown in Fig. 6.

Table II

PROTOTYPE

\begin{tabular}{ll|ll}
\hline \hline Component & Part/Value & Component & Part/Value \\
\hline \hline$V_{D D}$ & $5 \mathrm{~V}$ & DAS & Analog Discovery kit \\
Opamp & LF357 & $C$ & $1 \mathrm{nF}$ \\
microcontroller & ATMEGA328 & $R_{\text {ref }}$ & $50.09 \mathrm{k} \Omega$ \\
\hline \hline
\end{tabular}

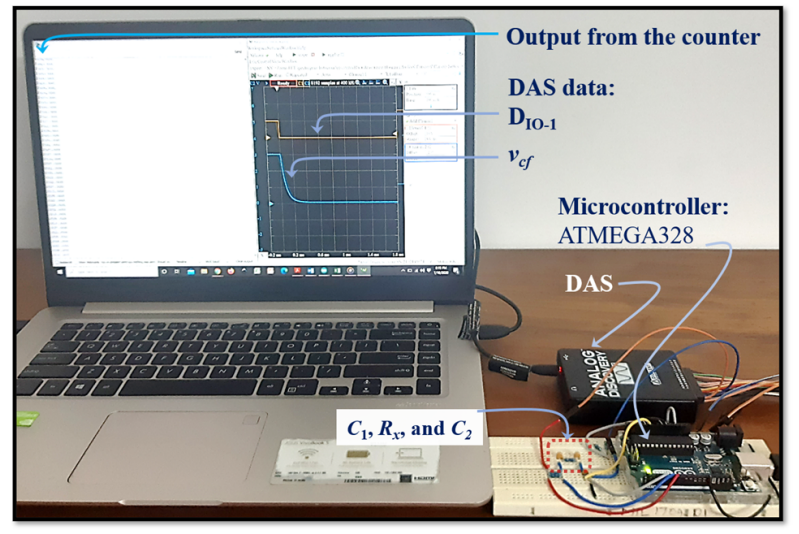

Fig. 6. Photograph of the experimental set-up.

\section{A. Measurement of $R_{x}$}

1) DAS-based Prototype

In order to assess the practicality of the measurement scheme, first an experimental set-up was developed in such a way that its performance was not limited by the non-idealities of the microcontroller. For this, the functionality of the proposed circuit given in Fig. 1(b) was realized in hardware with the help of a DAS.

First, the mode - $R_{x}$ was implemented. For this, the function generator was set to give a square wave (with a frequency of $200 \mathrm{~Hz}$, amplitude of $5 \mathrm{~V}$, and an offset of $2.5 \mathrm{~V}$ ) emulating the function of pin $\mathrm{D}_{\mathrm{IO}-1}$. In addition, $R_{\text {ref }}$ was shorted with a wire

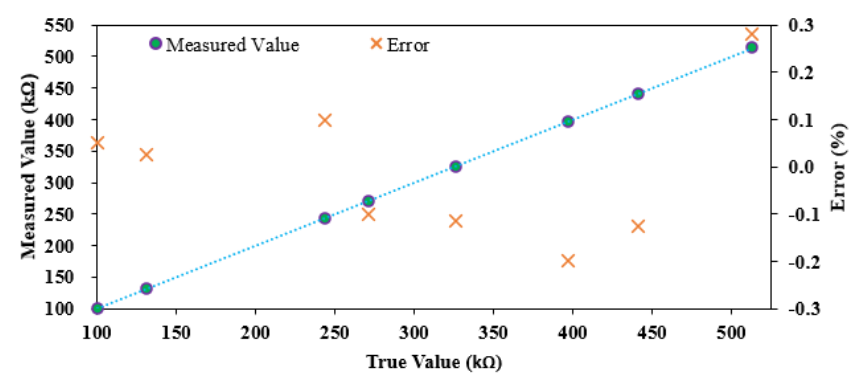

Fig. 7. Results from the prototype for a range of $R_{x}$. The output and the error obtained are shown.

in order to measure only $R_{x}$. Node ' $\mathrm{b}$ ' was connected to the input of a voltage follower, built using a very low bias current opamp (LF357), whose output was given to the DAS. The analog channels of the DAS were used to acquire the important waveforms. The connections to the DAS were made such that channel-1 captured the signal at $\mathrm{D}_{\mathrm{IO}-1}$, whereas channel-2 captured $v_{c f}$ at the output of the voltage follower. To operate in mode - $\left(R_{x}+R_{r e f}\right)$, the short across $R_{\text {ref }}$ was removed. The developed system enables performing mode $-R_{x}$ and mode - $\left(R_{x}+R_{r e f}\right)$ independently and acquires the corresponding $v_{c f}$ waveforms.

A VI was accordingly developed, using LabVIEW, to acquire the signals from the DAS and process them, as described further. For a chosen value of $R_{x}$, the $v_{c f}$ data during the discharge phase was analyzed. The VI was programmed to perform an exponential fit of the $v_{c f}$ data and provide the parameters representing the decaying exponential curve to compute $T_{1}$. For this computation, $V_{T L}=300 \mathrm{mV}$. Then, the $v_{c f}$ data corresponding to mode - $\left(R_{x}+R_{\text {ref }}\right)$ was taken and processed to compute $T_{2}$ for the same $V_{T L}$. Once $T_{1}$ and $T_{2}$ were computed, the value of $R_{x}$ was calculated by the VI using (7). This experiment was repeated for a range of $R_{x}$ from $100 \mathrm{k} \Omega$ to $500 \mathrm{k} \Omega$, in several steps. Later, this study was repeated for $50 \mathrm{k} \Omega, 700 \mathrm{k} \Omega$ and $800 \mathrm{k} \Omega$ and the accuracy was within the same range. The coupling capacitors used in this study were $C_{1}$ $=C_{2}=4 \mathrm{nF}$, thus resulting in $V_{C F}=3.3 \mathrm{~V}$. In each measurement, the actual value of $R_{x}$ was measured using a 6-1/2 digit multimeter (34410A, from Agilent Technologies) with an accuracy of $\pm(0.012 \%$ of the reading $+0.001 \%$ of the chosen range $)$. This was used to compute the error introduced by the proposed method. The results are shown in Fig. 7. The maximum error (expressed as a percentage of the full scale, $\% \mathrm{FS}$ ) noted during the experiments was $0.28 \%$, indicating the high accuracy of the proposed approach, although its simplicity.

As mentioned in section I, for the potential tap water conductivity monitoring in the range of 50-800 $\mu \mathrm{S} / \mathrm{cm}[12]$, the resistance of the water column of interest lies in the range between $50 \mathrm{k} \Omega$ and $750 \mathrm{k} \Omega$; this is assuming a distance between the electrodes of $12 \mathrm{~cm}$ and an inner diameter of the insulating tube of $6.35 \mathrm{~mm}$. The range can be adjusted by adjusting the distance between the electrodes. As described above, to study the performance of the proposed measurement scheme, the value of $R_{x}$ was incremented in several steps within that range. For low values of $R_{x}$ (say, lower than $1 \mathrm{k} \Omega$ ) with a coupling 


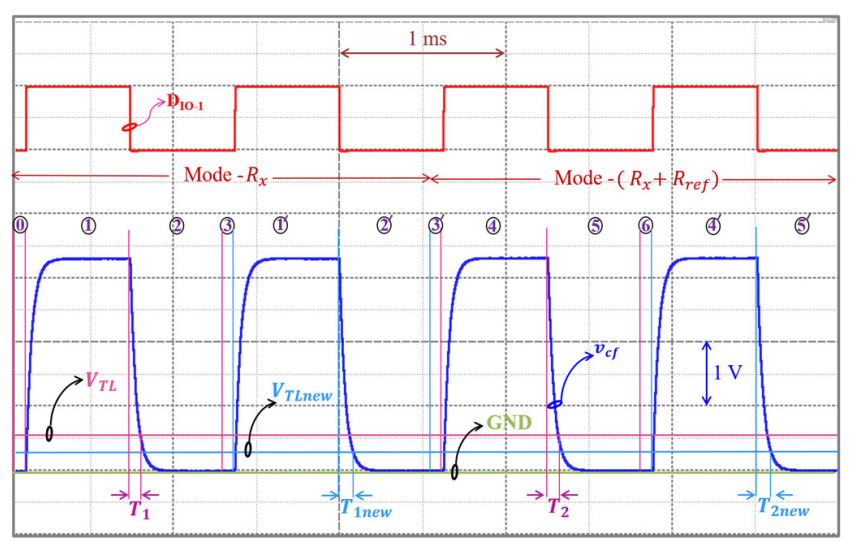

Fig. 8. $v_{c f}$ and $\mathrm{D}_{\mathrm{IO}-1}$ waveforms recorded, using DAS, from the prototype during the measurement process. States 0 to 6 correspond to $R_{x}$ measurement while 1', 2', 3', 4', and 5' correspond to the cycles used for $C_{12}$ measurement.

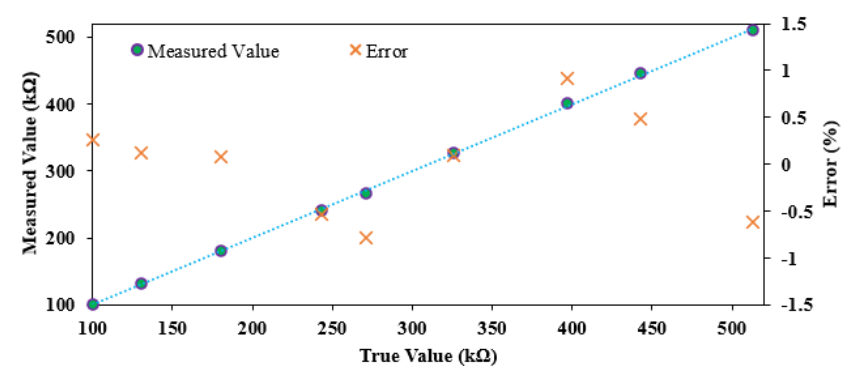

Fig. 9. Results from the prototype-2 for a range of $R_{x}$. This prototype was implemented using microcontroller.

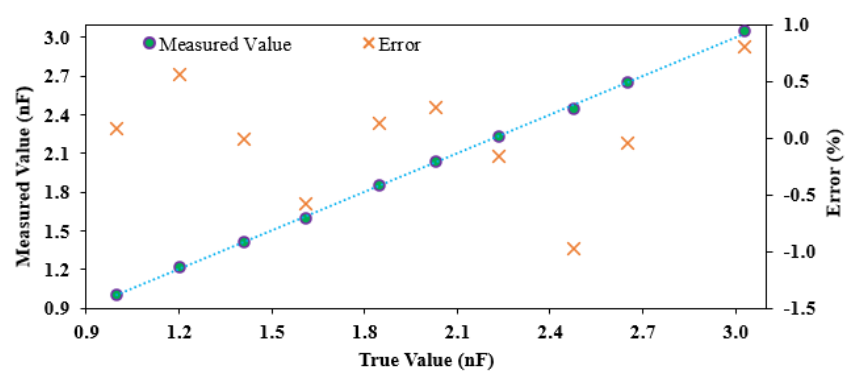

Fig. 10. Results from the DAS based prototype for a range of $C_{12}$.

capacitance of $2 \mathrm{nF}$ [1], the discharge time will be very short and the resolution of the timer embedded into the ATMEGA328 would not be sufficient to accurately measure it. Thus, for the proposed design using the ATMEGA328, the range of $R_{x}$ should be such that the discharge time is sufficiently long for the embedded timer to measure accurately. Once the range of $R_{x}$ is fixed, $R_{r e f}$ is chosen so that $\left(T_{2}-T_{1}\right)$ is much longer than the timer resolution. In addition, $R_{r e f}$ is set lower than $R_{x}$, to avoid an unnecessary increase of the overall measurement time. 2) Microcontroller-based Prototype

In this experiment, instead of using the DAS and the VI as before, the scheme was entirely realized using a microcontroller as given in Fig. 1(b), supplied at $5 \mathrm{~V}$. The signal $v_{c f}$ was given to node $\mathrm{A}_{1}$ of the microcontroller, and the built-in timer was programmed to measure, with a timing resolution of $62.5 \mathrm{~ns}$, the time intervals $T_{1}$ and $T_{2}$ during the corresponding states ' 2 ' and ' 5 ', respectively, as given in Table-I. The important waveforms were recorded using the oscilloscope in Analog Discovery kit, for the charge and discharge cycles for mode $R_{x}$ followed by mode - $\left(R_{x}+R_{\text {ref }}\right)$, as shown in Fig. 8; note that states 1 ' to 5 ' in Fig. 8 correspond to $C_{12}$ measurement presented later in section IV.B. The experiment was repeated for the same set of values of $R_{x}$ employed in section IV-A.1. The value of $R_{x}$ in each case, and the corresponding error in $\% \mathrm{FS}$, was computed and is presented in Fig. 9. The maximum error noted during the experiments was $0.91 \%$. The output of this experiment, in comparison with that from section IV-A.1, reflects the effect of the non-idealities of the components and ICs used in the microcontroller-based prototype.

\section{B. Measurement of $C_{12}$}

\section{1) DAS-based Prototype}

This testing approach is similar to the one used to prove the feasibility to measure $R_{x}$. In this study, the signal at $\mathrm{D}_{\mathrm{IO}-1}$ was generated using the function generator in the Analog Discovery kit, serving as DAS. This signal and $v_{c f}$ at node $b$ were recorded using the same DAS. This data was provided to the VI developed in section IV.A.1, to perform an exponential fit of the $v_{c f}$ data and provide the parameters. It was modified to compute the time intervals $T_{1}$ and $T_{1 \text { new }}$ for $V_{T L}=500 \mathrm{mV}$ and $V_{\text {TLnew }}=300 \mathrm{mV}$, respectively. Afterwards, using the same procedure, $T_{2}$ and $T_{2 n e w}$ were determined. Once the time values were computed, the value of $C_{12}$ was calculated by the VI using (14). In these tests, $R_{x}$ was kept constant at $100 \mathrm{k} \Omega$ and $C_{12}$ was varied in steps of $200 \mathrm{pF}$ within the range from 1 $\mathrm{nF}$ to $3 \mathrm{nF}$. The actual values were measured using an LCR meter (HP 4274A) that has an accuracy of $0.1 \%$. The results obtained are shown in Fig. 10. The maximum error, in full scale, noted during the experiments was $-0.96 \%$.

2) Microcontroller-based Prototype

To perform and verify the accuracy of the microcontrollerbased measurement of $C_{12}$, as presented in section II. B., the program for the prototype developed using the ATMega328 microcontroller to test the measurement of $R_{x}$ was modified. A new cycle was included in each of the modes, to measure $C_{12}$, along with $R_{x}$. Thus, now there are four time measurements, two in each of the two modes of operation. The operations of the digital pins $\mathrm{D}_{\mathrm{IO}-1}, \mathrm{D}_{\mathrm{IO}-2}$, and $\mathrm{D}_{\mathrm{IO}-3}$ are identical in both the cycles present in the same mode. The only change is in the threshold $\left(V_{\text {TLnew }}\right)$ used in the new cycles. The following modes were executed: mode- $R_{x}$ with $V_{T L}=500 \mathrm{mV}$, followed by the

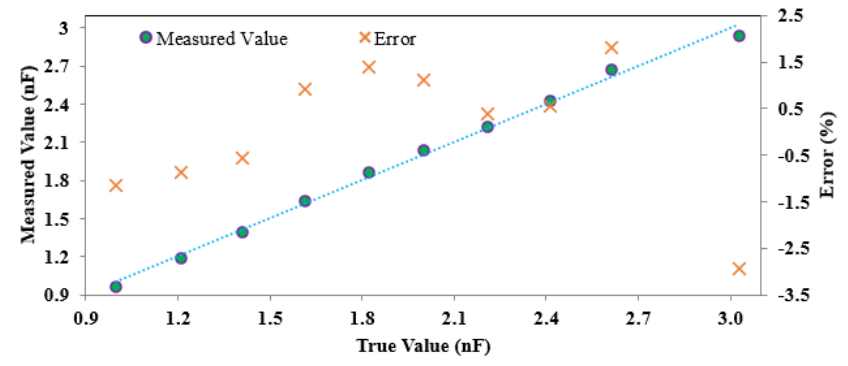

Fig. 11. Results from the microcontroller-based prototype, for the measurement of $C_{12}$. 
same mode with $V_{\text {TLnew }}=300 \mathrm{mV}$, and then mode- $\left(R_{x}+R_{\text {ref }}\right)$ with $V_{T L}=500 \mathrm{mV}$, followed by with $V_{\text {TLnew }}=300 \mathrm{mV}$, as shown in Fig. 8. This was repeated for the same range of $C_{12}$ as in the DAS-based prototype. From the measured discharging times, the value of $C_{12}$, in each case, was estimated using (14). The corresponding error in each measurement was computed, and presented in Fig. 11. As can be seen, the maximum error noted during the experiments was $-2.94 \%$ (full scale). Based on the results given in section IV.B.1, one can conclude that an improvement in the accuracy of the results is possible with a higher-end microcontroller.

\section{Noise and Resolution Study}

An experimental study was conducted to evaluate the noise and resolution in the measurements carried out by the microcontroller-based prototype. In this study, $R_{x}$ and $C_{12}$ were set to $100 \mathrm{k} \Omega$ and $1 \mathrm{nF}$, respectively, and 200 readings were recorded in a continuous manner. From this data, the signal to noise ratio (SNR), standard deviation (SD), $\sigma$, effective number of bits (ENOB), and repeatability, $d$, were computed using the formulae given in Table III and presented in the same table. Accordingly, a resolution of 8 bits is feasible for the measurement of both $R_{x}$ and $C_{12}$ which is a remarkable value considering the simplicity of the proposed circuit and the complexity of the sensor circuit model.

The repeatability experiment was carried out for several resistance values between $50 \mathrm{k} \Omega$ and $800 \mathrm{k} \Omega$. The output was

TABLE III

NOISE AND RESOLUTION STUDY

\begin{tabular}{lcc}
\hline \hline Parameter and Formula & $R_{x}$ & $C_{12}$ \\
\hline \hline SNR $=10 \log \frac{\sum_{i=1}^{M} X(i)^{2}}{\sum_{i=1}^{M}[X(i)-\bar{X}]^{2}}$ & $50.47 \mathrm{~dB}$ & $51.1 \mathrm{~dB}$ \\
Standard deviation, $\sigma=\sqrt{\frac{\sum_{i=1}^{M}[X(i)-\bar{X}]^{2}}{M-1}}$ & $0.30 \mathrm{k} \Omega$ & $5.94 \mathrm{pF}$ \\
ENOB $=\frac{(S N R-1.76)}{6.02}$ & $8.1 \mathrm{bits}$ & $8.2 \mathrm{bits}$ \\
Repeatability, $d=\frac{\Delta_{r \max }}{X_{u}-X_{l}} \times 100 \%$ & $0.51 \%$ & $1.13 \%$ \\
\hline \hline
\end{tabular}

$X(i)=i^{\text {th }}$ measurement; $\bar{X}=$ average value of the measured data

$M=$ total number of measurements

$X_{u}, X_{l}=$ upper and lower limits of the measured data

$\Delta_{\text {rmax }}=$ maximum difference between repeated measurements

TABLE IV

STANDARD DEVIATION (SD) FOR A WIDE RANGE OF $R$

\begin{tabular}{|c|c|c|c|c|c|c|c|c|c|}
\hline \hline$R_{x}[\mathrm{k} \Omega]$ & 50 & 100 & 200 & 300 & 400 & 500 & 600 & 700 & 800 \\
\hline $\mathrm{SD}[\mathrm{k} \Omega]$ & 0.303 & 0.303 & 0.304 & 0.305 & 0.304 & 0.305 & 0.305 & 0.301 & 0.305 \\
\hline
\end{tabular}

TABLE V

SD FOR $100 \mathrm{k} \Omega$ MEASUREMENT AT 12 INTERVALS

\begin{tabular}{|c|c|c|c|c|c|c|}
\hline \hline Time $[\mathrm{hr}]$ & 1 & 2 & 3 & 4 & 5 & 6 \\
\hline $\mathrm{SD}[\mathrm{k} \Omega]$ & 0.302 & 0.301 & 0.304 & 0.302 & 0.304 & 0.304 \\
\hline Time $[\mathrm{hr}]$ & 7 & 8 & 9 & 10 & 11 & 12 \\
\hline $\mathrm{SD}[\mathrm{k} \Omega]$ & 0.304 & 0.305 & 0.303 & 0.300 & 0.301 & 0.301 \\
\hline \hline
\end{tabular}

recorded for a data length of a hundred measurements, for each resistor, and the corresponding SD was computed. As presented in Table IV, SD was very similar for the different resistance values under test. Later, the experiment was continued for the $100 \mathrm{k} \Omega$ case for $12 \mathrm{hrs}$, with the aim of testing any potential time drifts. In this, the microcontroller was powered all the time and a hundred measurements recorded for every one hour. The corresponding computed SD is presented in Table V. Again, the values of $\mathrm{SD}$ of the measured outputs remained very similar through the course of the day.

\section{DISCUSSION}

When a series combination of resistance and capacitance needs to be measured, a widely used method, apart from the conventional bridge-based one [6] that requires manual balancing, is synchronous demodulation [24]. This requires a precision sine wave excitation, multipliers, low-pass filters, followed by a digitizer and a processor for computation. A slightly optimized version of this mechanism is available in [25]. It is still complex, as it requires an on-board digital signal processing engine to compute discrete Fourier transform of the digitized signal. The synchronous demodulation approach gives high accuracy, but it is complex considering the number of individual units required to implement the analog multiplication, and the overall power consumption. A scheme based on a modified Martin oscillator is presented in [26] for impedance measurement. This requires a precision current source. The oscillator has to be operated in four different modes, and the values of resistance and capacitance are computed from the four oscillator time periods measured, one from each mode. This method is complex and time-consuming compared to the direct-microcontroller approach presented in this paper. On the other hand, there are several schemes available for the measurement of capacitance [27], [28] and resistance [6], [29] but none of these are useful to measure the resistance of a capacitively-coupled resistive sensor.

In the literature, there are direct-microcontroller interfaces for the measurement of a single component: resistance in [15][18], [30], [31], [32], capacitance in [19], [20], and inductance in [21], [22]. In [33] both resistance and capacitance were measured, but for a lossy capacitive sensor where resistance and capacitance are in parallel.

The capacitively-coupled resistive sensor has not been explored so far from the perspective of designing a directmicrocontroller interface. A previous work [23] by the authors deals with a suitable circuit for such a sensor, but it was designed to measure only the resistance. In some applications, however, the value of the capacitance that is in series with the resistive sensor carries useful information. Therefore, it will be advantageous if the same interface could measure both resistance and capacitance. In this context, it is for the first time that a direct-microcontroller interface able to measure both components in a capacitively-coupled resistive sensor has been developed and evaluated. The proposed method of estimating the coupling capacitance $C_{12}$ does not require any additional hardware compared to the initial study presented in [23]. Since 
TABLE VI

COMPARISON

\begin{tabular}{|c|c|c|c|c|c|}
\hline Ref. & \multicolumn{2}{|c|}{ Type of sensor } & $\begin{array}{l}\text { Conversi- } \\
\text { on time }\end{array}$ & $\begin{array}{l}\text { Resolu- } \\
\text { tion }\end{array}$ & $\begin{array}{l}\text { Accura } \\
- \text { cy }[\%]\end{array}$ \\
\hline$[15]$ & \multicolumn{2}{|c|}{ Single resistive sensor } & $\approx 50 \mathrm{~ms}$ & $0.1 \Omega$ & $0.85^{(\mathrm{a})}$ \\
\hline [17] & \multicolumn{2}{|c|}{$\begin{array}{l}\text { Single resistive sensor } \\
\text { with lead wire resistance }\end{array}$} & $2.58 \mathrm{~ms}$ & $0.12 \Omega^{(\mathrm{b})}$ & $0.59^{(\mathrm{c})}$ \\
\hline [19] & \multicolumn{2}{|c|}{ Single capacitive sensor } & $1.5 \mathrm{~s}$ & NS & 0.8 \\
\hline [20] & \multicolumn{2}{|c|}{$\begin{array}{c}\text { Differential capacitive } \\
\text { sensor }\end{array}$} & $50 \mathrm{~ms}$ & 7 bits & $1.1^{(\mathrm{d})}$ \\
\hline [33] & \multicolumn{2}{|c|}{$\begin{array}{l}\text { Resistance and } \\
\text { capacitance connected } \\
\text { in parallel }\end{array}$} & $\approx 3 \mathrm{~ms}$ & NS & 1 \\
\hline \multirow{2}{*}{$\begin{array}{l}\text { This } \\
\text { work }^{(e)}\end{array}$} & \multirow{2}{*}{$\begin{array}{l}R_{x} \text { and } C_{12} \\
\text { connected in } \\
\quad \text { series }\end{array}$} & $R_{\mathrm{x}}$ & $5 \mathrm{~ms}$ & 8.1 bits & $0.91^{(f)}$ \\
\hline & & $C_{12}$ & $5 \mathrm{~ms}$ & 8.2 bits & $2.94^{(\mathrm{f})}$ \\
\hline \multicolumn{6}{|c|}{$\begin{array}{l}\text { (a) After a calibration, it was reduced to } 0.01 \% .{ }^{(\text {b) }} \text { Standard deviation. } \\
{ }^{\text {(c) }} \text { Hysteresis error. NS-Not studied. }{ }^{(\mathrm{d})} \text { Nonlinearity error. } \\
\text { (e) }[23] \text { deals } \\
\text { with } R_{x} \text { alone, not measurement of } C_{12 .}{ }^{(\mathrm{f})} 0.28 \% \text { (resistance) and } 0.96 \% \\
\text { (capacitance) for DAS-based method. }\end{array}$} \\
\hline
\end{tabular}

the direct microcontroller interface is simple, robust, low-cost, and consumes less power [34], it offers the opportunity to realize portable and scalable measurement systems that can be widely used for this type of sensor.

As presented in Table VI, the new scheme offers a performance which is almost on par with the schemes (without special calibration) available to individually measure resistance and capacitance, whilst providing a direct-microcontroller method to measure both when connected in series. This functionality is achieved without additional components or parts in the measurement system.

\section{CONCLUSION}

The proposed direct microcontroller interface for capacitively-coupled resistive sensors, measuring both the resistance and the coupling capacitance, has been attempted for the first time. The measured resistance output is independent of the values of the coupling capacitances, the charging capacitor, the dc excitation voltage, and the preset threshold voltage. When a resistive sensor is coupled to the measurement system through capacitances, one of the main challenges is to make the resistance measurement insensitive to variations in the coupling capacitances, which is achieved herein without using the synchronous demodulation technique adopted by conventional approaches. The proposed circuit is simple in design and completes both measurements in $10 \mathrm{~ms}$. The feasibility study of the proposed circuit for resistance and coupling capacitance measurement, exhibits a maximum error of $0.28 \%$ and $0.96 \%$, respectively, when evaluated without considering the nonidealities of the microcontroller. In the practical setup, maximum error noted for resistance measurement is $0.91 \%$, and that for the coupling capacitance is $2.94 \%$. Higher accuracy can be achieved by selecting a microcontroller with more appropriate specifications. The proposed scheme uses only a simple microcontroller and a few passive components and hence can realize a low-cost, low-power measurement system.
Owing to these attractive features, the proposed measurement circuit is expected to be extensively used in non-intrusive sensing and monitoring applications based on capacitivelycoupled resistive sensors.

\section{REFERENCES}

[1] K. K. Tejaswini, B. George, V. J. Kumar, R. Srinivasan and S. Tata, "A Capacitive Coupled Non-Contact Probe for the Measurement of Conductivity of Liquids, " IEEE Trans. Instrum. Meas., vol. 68, no. 5, pp. 1602-1610, May 2019.

[2] A. Arthor, The Electrical Conductivity of Aqueous Solutions. New York, NY, USA: Andesite Press, 2017.

[3] J. T. Griffin, "Touch-sensitive display with capacitive and resistive touch sensors and method of control," U.S. Patent application US 8179376, May, 2012.

[4] Y. Xue, Z. Ju, K. Xiang, J. Chen and H. Liu, "Multimodal Human Hand Motion Sensing and Analysis - A Review," IEEE Trans. Cogn. Devel. Syst., vol. 11, no. 2, pp. 162-175, June 2019.

[5] L. Pan, A. Chortos, G. Yu, S. Isaacson, R. Allen, Y. Shi, R. Dauskardt and Z. Bao, "An ultra-sensitive resistive pressure sensor based on hollow-sphere microstructure induced elasticity in conducting polymer film," Nature Commun., vol. 5, pp. 3002, Jan. 2014.

[6] L. Paredes-Madrid, J. Fonseca, A. Matute, E. Gutiérrez Velásquez and C. Palacio, "Self-compensated driving circuit for reducing drift and hysteresis in force sensing resistors," Electronics, Aug 2018.

[7] A. Song, L. Fu, "Multi-dimensional force sensor for haptic interaction: A review," Virtual Reality Intell. Hardw., vol. 1, no. 2, pp. 121-135, Jan. 2019.

[8] E. O. Doebelin, Measurement Systems-Application and Design, 5th ed. New York, NY, USA: McGraw-Hill, 2004.

[9] E. Al Hosani, M. Zhang and M. Soleimani, "A Limited Region Electrical Capacitance Tomography for Detection of Deposits in Pipelines," IEEE Sensors J., vol. 15, no. 11, pp. 6089-6099, Nov. 2015.

[10] S. L. Schiefelbein, N. A. Fried, K. G. Rhoads and D. R. Sadoway, "A high-accuracy, calibration-free technique for measuring the electrical conductivity of liquids," Review of scientific instruments, vol. 28, no. 6 , pp. 1141-1149, Dec. 1998.

[11] B. Wang, W. Tan, Z. Huang, H. Ji, and H. Li, "Image reconstruction algorithm for capacitively coupled electrical resistance tomography," Flow Meas. Instrum., vol. 40, pp. 216-222, 2014.

[12] Fondriest Environmental, Inc., "Conductivity, Salinity and Total Dissolved Solids." Fundamentals of Environmental Measurements, [Online]. Available: https://www.fondriest.com/environmentalmeasurements/parameters/water-quality/conductivity-salinity-tds/

[13] M. V. Storey, B. Van der Gaag, and B. P. Burns, "Advances in on-line drinking water quality monitoring and early warning systems", Water research, 45(2), pp.741-747, 2011.

[14] W. H. Clements and C. Kotalik, "Effects of major ions on natural benthic communities: an experimental assessment of the US Environmental Protection Agency aquatic life benchmark for conductivity", Freshwater Science, 35(1), pp. 126-138, 2016.

[15] F. Reverter, J. Jordana, M. Gasulla, and R. Pallàs-Areny, "Accuracy and resolution of direct resistive sensor-to-microcontroller interfaces," Sens. Actuators A, Phys., vol. 121, no. 1, pp. 78-87, 2005.

[16] E. Sifuentes, R. Gonzalez-Landaeta, J. Cota-Ruiz, and F. Reverter, "Seat occupancy detection based on a low-power microcontroller and a single FSR, " Sensors, vol. 19, no. 3, 699 (11pp), Feb. 2019.

[17] R. Anandanatarajan, U. Mangalanathan, and U. Gandhi, "Enhanced microcontroller interface of resistive sensors through resistance-to-time converter, " IEEE Trans. Instrum. Meas., vol. 69, no. 6, pp. 2698-2706, June 2020.

[18] P. R. Nagarajan, B. George and V. J. Kumar, "Improved Single-Element Resistive Sensor-to-Microcontroller Interface," IEEE Trans. Instrum. Meas., vol. 66, no. 10, pp. 2736-2744, Oct. 2017.

[19] J. E. Gaitan-Pitre, M. Gasulla and R. Pallas-Areny, "Analysis of a Direct Interface Circuit for Capacitive Sensors," IEEE Trans. Instrum. Meas., vol. 58, no. 9, pp. 2931-2937, Sept. 2009.

[20] F. Reverter and Ò. Casas, "Interfacing Differential Capacitive Sensors to Microcontrollers: A Direct Approach," IEEE Trans. Instrum. Meas., vol. 59 , no. 10, pp. 2763-2769, Oct. 2010.

[21] N. Ramadoss, and B. George, "A simple microcontroller based digitizer for differential inductive sensors," Proc. of 2015 IEEE International 
Instrumentation and Measurement Technology Conference ( $\left.I^{2} M T C\right)$, May 11, 2015, pp. 148-153.

[22] Z. Kokolanski, J. Jordana, M. Gasulla, V. Dimcev, and F. Reverter, "Direct inductive sensor-to-microcontroller interface circuit," Sens. Actuators A Phys., vol. 224, pp. 185-191, 2015.

[23] L. Areekath, B. George, and F. Reverter, "A Simple Direct Microcontroller Interface for Capacitively-Coupled Resistive Sensors," Proc. of 2020 IEEE International Instrumentation and Measurement Technology Conference ( $\left.I^{2} M T C 2020\right)$, May 25-28, 2020, Dubrovnik, Croatia, 2020, pp. 1-5.

[24] R. Pallas-Areny and J. G. Webster, Sensors and Signal Conditioning. New York, NY, USA: Wiley, 2001.

[25] Analog Devices, Norwood, MA, USA. (2013). Datasheet, AD5933, 1 MSPS, 12-Bit Impedance Converter, Network Analyzer. [Online] Available:http://www.analog.com/static/importedfiles/data sheets/AD5 933.pdf

[26] S. N. Nihtianov, G. P. Shterev, B. Iliev and G. C. M. Meijer, "An interface circuit for R-C impedance sensors with a relaxation oscillator," in IEEE Transactions on Instrumentation and Measurement, vol. 50, no. 6, pp. 1563-1567, Dec. 2001

[27] Á. Pintér, I. Dénes, "Interface circuit for measuring small capacitance changes in sensor networks," IET Science, Measurement \& Technology, vol. 9 , no. 5, pp. 570-578, July 2015.

[28] L. Areekath, B. George and F. Reverter, "An Extended Study on an Interference-Insensitive Switched Capacitor CDC," IEEE Sensors J., vol. 19, no. 18, pp. 8283-8292, Sept.15, 2019.

[29] P. M. Ramos, J. M. D. Pereira, H. M. G. Ramos, and A. L. Ribeiro, "A four-terminal water-quality-monitoring conductivity sensor," IEEE Trans. Instrum. Meas., vol. 57, no. 3, pp. 577-583, Mar. 2008.

[30] J. A. Hidalgo-López, J. A. Botín-Córdoba, J. A. Sánchez-Durán, J. C. Tejero-Calado and Ó. Oballe-Peinado, "Improved Calibration Method for Resistive Sensors using Direct Interface Circuits," IEEE Trans. Instrum. Meas., DOI: 10.1109/TIM.2019.2958583.

[31] J.A. Hidalgo-López, J.A. Botín-Córdoba, J.A. Sánchez-Durán and O. Oballe-Peinado, "Fast calibration methods for resistive sensor readout based on direct interface circuits, " Sensors, vol. 19, no. 18, 3871 (20pp), Sep. 2019.

[32] Z. Czaja, "An implementation of a compact smart resistive sensor based on a microcontroller with an internal adc", Metrology and Measurement Systems, vol. 23, no. 2, pp. 225-238, 2016.

[33] F. Reverter, O. Casas, "A microcontroller-based interface circuit for lossy capacitive sensors", Measurement Science and Technology, 21(6), p.065203, May, 2010.

[34] F. Reverter, "Power Consumption in Direct Interface Circuits," IEEE Trans. Instrum. Meas., vol. 62, no. 2, pp. 503-509, Feb. 2013. 